\title{
Environmental exposures in young adults with declining kidney function in a population at risk of Mesoamerican Nephropathy
}

Evangelia-Theano Smpokou, Marvin González-Quiroz, Carla Martins, Paula Alvito, Jennifer

Le Blond, Jason Glaser, Aurora Aragón, Catharina Wesseling, Dorothea Nitsch, Neil Pearce, Jill Norman, Christian Lindh, Jackie Morton and Ben Caplin

Evangelia-Theano Smpokou, MSc. Department of Renal Medicine, Division of Medicine, University College London, London, UK. evangelia.smpokou.13@ucl.ac.uk

Marvin González-Quiroz, PhD. Research Centre on Health, Work and Environment (CISTA), National Autonomous University of Nicaragua at León (UNAN-León), León, Nicaragua. Department of Non-Communicable Disease Epidemiology, London School of Hygiene and Tropical Medicine, London, UK. Department of Renal Medicine, Division of Medicine, University College London, London, UK. marvin.gonzalez@1shtm.ac.uk or marvin99_00@yahoo.es

Carla Martins, BSc. National Institute of Health Dr. Ricardo Jorge (INSA), Lisboa, Centre for Environmental and Marine Studies (CESAM), University of Aveiro, Aveiro, Portugal. NOVA National School of Public Health, Universidade NOVA de Lisboa, Lisboa, Portugal. carla.martins@insa.min-saude.pt

Paula Alvito, PhD. National Institute of Health Dr. Ricardo Jorge (INSA), Lisboa, Portugal. Centre for Environmental and Marine Studies (CESAM), University of Aveiro, Aveiro. paula.alvito@insa.min-saude.pt

Jennifer Le Blond, PhD. Royal School of Mines, Imperial College London, London, UK. jennifer.leblond@gmail.com

Jason Glaser, BSc. La Isla Network, Washington DC, USA. London School of Hygiene and Tropical Medicine, London, UK. jason@laislanetwork.org 
Aurora Aragón, PhD. Research Centre on Health, Work and Environment (CISTA), National Autonomous University of Nicaragua at León (UNAN-León), León, Nicaragua. aurora.aragon@cm.unanleon.edu.ni

Catharina Wesseling, PhD. Institute of Environmental Medicine, Karolinska Institutet, Stockholm, Sweden. La Isla Network, Washington DC, USA. inekewesseling@gmail.com

Dorothea Nitsch, D.Med. Department of Non-Communicable Disease Epidemiology, London School of Hygiene and Tropical Medicine, London, UK. dorothea.nitsch@1shtm.ac.uk

Neil Pearce, PhD. Departments of Medical Statistics and Non-Communicable Disease Epidemiology, Centre for Global NCDs, London School of Hygiene and Tropical Medicine, London, UK. neil.pearce@1shtm.ac.uk

Jill Norman, PhD. Department of Renal Medicine, Division of Medicine, University College London, London, UK. j.norman@ucl.ac.uk

Christian Lindh, PhD. Division of Occupational and Environmental Medicine, Lund University, Lund, Sweden.christian.lindh@med.lu.se

Jackie Morton, PhD. Biological Monitoring, Health and Safety Executive, Harpur Hill, Buxton, Derbyshire, UK. jackie.morton@hse.gov.uk

Ben Caplin, PhD. Department of Renal Medicine, Division of Medicine, University College London, London, UK. b.caplin@ucl.ac.uk 
Key words: Chronic Kidney Disease of unknown origin (CKDu), nephrotoxicity, pesticides, Mesoamerican Nephropathy (MeN), environmental toxins

Correspondence to: Evangelia-Theano Smpokou, Department of Renal Medicine,

University College London, Royal Free Campus, Rowland Hill Street, London NW3 2PF,

UK. evangelia.smpokou.13@ucl.ac.uk

\section{Word Count}

Abstract: 250

Main body: 2970 


\begin{abstract}
Objectives: There is an epidemic of Mesoamerican Nephropathy (MeN) in Central America, where sugarcane production is prominent. Numerous causes are proposed, but to date limited evidence supports any one hypothesis. A nested case-control study using biosamples from a rural, communitybased follow-up study of 350 young adults from Northwest Nicaragua at risk of MeN, was conducted with the aim of characterising the associations between urinary concentrations of metals, pesticides and mycotoxins from samples collected in the first 6 months, and decline in kidney function over 2 years.
\end{abstract}

Methods: Urine samples collected at baseline (pre-sugarcane harvest) and the first 6-month follow-up (post-sugarcane harvest) visit were tested. Twelve metals and metalloids ( $\mathrm{Al}$, total $\mathrm{As}, \mathrm{Cd}, \mathrm{Co}, \mathrm{Cr}, \mathrm{Cu}$, $\mathrm{Hg}, \mathrm{Mn}, \mathrm{Pb}, \mathrm{Se}, \mathrm{Si}$ and $\mathrm{Sr}$ ) were analysed by inductively-coupled plasma-mass spectrometry. Twelve pesticides or their metabolites (2,4-D, 3-PBA, 4F3PBA, CFCA, DCCA, ETU, glyphosate, MCPA, OH-PYM, 5-OH-TBZ, TEB-OH and TCP), and two mycotoxins (OTA and CIT), were analysed by liquid chromatography coupled mass spectrometry. Differences in the creatinine-corrected urinary concentrations of the measured exposures between outcome groups (participants with stable versus declining kidney function) were examined.

Results: Elevated levels of aluminium and total arsenic as well as metabolites of several pesticides were detected across the population. No differences were identified between the declining and stable groups in the levels of metals or pesticides tested. OTA and CIT were below the limit of detection. Conclusions: The tested metals, metalloids, pesticides and mycotoxins were not associated with loss of kidney function in participants at-risk of MeN. 


\section{Key messages}

\section{What is already known about this subject?}

- Mesoamerican Nephropathy (MeN); also known as Chronic Kidney Disease of undetermined aetiology $(\mathrm{CKDu})$, has affected mainly agricultural workers; particularly sugarcane cutters, in the rural areas of Nicaragua, El Salvador, Guatemala and Costa Rica over the last 3 decades.

- Proposed causes of CKDu include occupational and environmental exposures, including food and water contaminated with metals, pesticides (in particular glyphosate) and possibly mycotoxins, as well as exposure to recurrent dehydration/heat.

\section{What are the new findings?}

- The population is exposed to elevated concentrations of aluminium and total arsenic but there was no association with renal decline.

- The levels of pesticide metabolites 3-PBA, DCCA and TCP indicate a degree of exposure of the population to pyrethroids and chlorpyrifos, whereas glyphosate was only detected infrequently and at low levels and there was no evidence of exposure to Ochatoxin A or Citrinin.

\section{How might this impact on policy or clinical practice in the foreseeable future?}

- Studies should investigate other potential causes of the disease including alternative environmental toxins, infectious diseases, response to dehydration/heat and potential genetic susceptibility.

- Beyond established occupational and environmental safety standards for the handling of pesticides, no additional interventions to prevent disease caused by the measured exposures can currently be recommended. 


\section{Introduction}

Over the last three decades, an unexplained form of chronic kidney disease (CKD) has emerged throughout Central America (1). The disease is known to predominantly affect people working in physically intense manual labour, especially those engaged in sugarcane cultivation and production (2). In Mesoamerica, it has been estimated that, by 2012, CKD of undetermined aetiology (CKDu also known as Mesoamerican Nephropathy $(\mathrm{MeN})$ ) had caused the premature death of at least 20,000, mainly male, young adults (3). Several similar epidemics of CKDu have been described in agricultural workers in other tropical regions, for example, Sri Lanka and India $(4,5)$.

Like many forms of $\mathrm{CKD}, \mathrm{MeN}$ is asymptomatic in the early stages. The key laboratory finding is increased serum creatinine ( $\mathrm{sCr}$ ) (reflecting a low estimated glomerular filtration rate; eGFR) which is observed before symptoms develop (6). Although both sexes are affected, the predominance of male agricultural workers suggests that occupational risk factors may play a major role in disease pathogenesis $(7,8)$.

To date, no clear cause of MeN has been identified. Environmental exposures to metals/metalloids, pesticides or mycotoxins, through contamination of water, soil, air and food could expose the population to hazardous levels (9). A number of metals, such as cadmium (Cd) and lead $(\mathrm{Pb})$, are known to be nephrotoxic (10). There are limited epidemiologic data relating chronic exposure to pesticides to CKD and most studies have found little supportive evidence for a role of agrochemicals in the development of MeN (11). Ochratoxin A (OTA) and citrinin (CIT) which frequently co-occurs with OTA, are nephrotoxins produced by fungi of the genera Aspergillus and Penicillium under high temperatures and humidity, can commonly be found in food, well water and animal feed (12)(13). OTA has been implicated as a causal agent in a number of endemic nephropathies, including chronic interstitial nephritis in Tunisia $(14,15)$.

Since 2014, our team has been conducting the first prospective community-based cohort study investigating the evolution and aetiology of MeN. The study initially recruited 350 healthy men and women (3:1 ratio), from 9 rural communities in north-western Nicaragua (16). Participants were 
invited to follow-up visits every 6 months (before and immediately after the sugarcane harvest). A rapid decline in kidney function was observed in approximately $10 \%$ of males and $3 \%$ of women over the first 2 years (16).

The objective of the present study was to determine the concentrations of various metals and metalloids, pesticides or their metabolites and mycotoxins in urine samples. Analysis was performed in samples collected at baseline and at the first 6 month follow-up visit and differences in the urinary concentration of exposures, between the group that experienced decline of kidney function and those with stable function over the 2 year study period, examined using a nested case-control approach.

\section{Methods}

\section{Study Population}

The study rationale, design and baseline findings have been reported elsewhere $(16,17)$. Briefly this was a population-representative, community-based follow-up study recruiting 350 men and women (ratio 3:1) aged 18-30 years without self-reported CKD or traditional CKD risk factors (diabetes or hypertension). Analyses were performed on urine samples collected at baseline (before the harvest visit 1) and 6 months later (at the end of the harvest - visit 2).

\section{Sample collection}

First morning urine samples (typically more concentrated and hence likely to contain higher levels of the analytes of interest) were collected between 3-6 am (before workers go to the fields). Samples were aliquoted into BD Vacutainers with boric acid $(2.63 \mathrm{mg} / \mathrm{mL}$ boric acid, $3.95 \mathrm{mg} / \mathrm{mL}$ sodium borate and $1.65 \mathrm{mg} / \mathrm{mL}$ sodium formate) for metal and metalloid analysis, and without boric acid for pesticide and mycotoxin analysis (see Supplementary Methods for details). Urinary biosamples were selected because they are relatively easy to collect and non-invasive. Samples were collected at the baseline visit (November 2014) and from the same individuals at the first follow-up visit 6 months later. 


\section{Outcome measure}

We used the same outcome groups that were derived as part of original analysis of the cohort followup (17). Briefly, a growth mixture model was used to agnostically allocate participants to renal function trajectory groups: (i) stable; (ii) rapid decline from a normal baseline eGFR; and (iii) established kidney dysfunction based on the eGFR calculated using serum creatinine and cystatin C (eGFR $\left._{\text {Scr-Scys }}\right)$ across the whole 2-year study period (visits 1 to 5).

As the aim was to investigate possible causal associations, we primarily examined the levels of exposures in the group of participants experiencing rapid decline in kidney function from a normal baseline (i.e. cases; $\mathrm{n}=25 \mathrm{men}$, with a mean change in eGFR $-18.2 \mathrm{~mL} / \mathrm{min} / 1.73 \mathrm{~m}^{2}$ per year) as compared to the levels in the stable group (i.e. controls; $n=213$ men, with a mean change in eGFR $-0.6 \mathrm{~mL} / \mathrm{min} / 1.73 \mathrm{~m}^{2}$ per year; including either the whole group or an age-, community- and occupation-matched sample). Comparisons of exposure concentrations in women, between the small number demonstrating a rapid decline $\left(\mathrm{n}=3\right.$, with a mean change in eGFR $-14.6 \mathrm{~mL} / \mathrm{min} / 1.73 \mathrm{~m}^{2}$ per year) and the stable group $\left(\mathrm{n}=84\right.$, with a mean change in eGFR $-0.6 \mathrm{~mL} / \mathrm{min} / 1.73 \mathrm{~m}^{2}$ per year), are presented in the Supplementary Material.

\section{Urinary analysis of metals, pesticides and mycotoxins}

All urine samples were analysed for elemental concentrations. Three hundred fifty samples from visit 1 and 291 samples from visit 2 (17\% of the participants lost to follow-up at visit 2$)$ were analysed by inductively-coupled plasma-mass spectrometry (ICP-MS) to measure aluminium ( $\mathrm{Al}$ ), total arsenic (As), cadmium $(\mathrm{Cd})$, chromium $(\mathrm{Cr})$, cobalt $(\mathrm{Co})$, copper $(\mathrm{Cu})$, lead $(\mathrm{Pb})$, manganese $(\mathrm{Mn})$, mercury $(\mathrm{Hg})$, selenium $(\mathrm{Se})$, silicon $(\mathrm{Si})$ and strontium $(\mathrm{Sr})$ in all study participants (see Supplementary Methods for further details). Levels of metals and metalloids were compared with thresholds reported in studies in Belgian, Mexican American and UK general population (18-21) (Supplementary Table and Figures).

Pesticides or their metabolites (selected because of evidence of local use along with analytical capability), OTA and CIT were measured in all the samples from men with rapidly declining kidney 
function and a number of age-, sex-, community- and occupation-matched individuals from the stable groups. In total, 57 samples from visit 1 and 46 samples from the same individuals from visit 2 were analysed by liquid chromatography-tandem mass spectrometry (LC-MS/MS) for (i) the phenoxyacid herbicides 2,4-dichlorophenoxyacetic acid (2,4-D) and 4-chloro-2-methylphenoxy acetic acid (MCPA), and the herbicide N-(phosphonomethyl)glycine (glyphosate); (ii) metabolites of insecticides; chloro-3,3,3-trifluoro-1-propen-1-yl-2,2-dimethylcyclopropanecarboxylic acid (CFCA), 3,5,6-trichloro-2-pyridinol (TCP), 4-fluoro-3-phenoxybenzoic acid (4F3PBA), 3-phenoxybenzoic acid (3-PBA) and cis/trans 3-(2,2-dichlorovinyl)-2,2-dimethylcyclopropane carboxylic acid (DCCA), all pyrethroid metabolites except TCP which is a metabolite of the organophosphate chlorpyrifos; and (iii) metabolites of conazole fungicides; hydroxy-tebuconazole (TEB-OH) and 5-hydroxytiabendazole (5-OH-TBZ), and metabolite of pyrimidine fungicides 3-hydroxy-pyrimetanil (OHPYR), and of dithiocarbamate fungicides, ethylenethiourea (ETU) (22-24) (see Supplementary Methods for further details).

OTA and CIT were determined by LC-MS/MS at the National Institute of Health Dr Ricardo Jorge, Portugal $(25,26)$ (see Supplementary Methods for further details).

To control for variation in urinary concentration levels of metals, metalloids, pesticides and mycotoxins were adjusted for creatinine (Jaffe reaction, see Supplementary Methods) (27).

\section{Statistical analyses}

Data were analysed using IBM SPSS Statistics version 25 with significance threshold set at $\mathrm{p}=0.05$. Descriptive analysis was undertaken separately for men and women. Unpaired student's t-test was used to test the null hypothesis that there were no differences in the parameter of interest and the kidney function trajectory groups in men only. Calculation of the effect size for the assessment of the power of the effect of the different kidney groups was performed using $G^{*}$ power 3.0.10 (Supplementary Methods). 


\section{Results}

The study population consisted of 263 men and 87 women recruited from nine communities (Table 1). At the beginning of the study $66 \%$ of the participants $(n=198)$ worked in agriculture including sugarcane and banana plantations.

The median creatinine-adjusted urinary concentrations and interquartile ranges of metals and metalloids from the whole cohort, for visit 1 and visit 2, men and women, are shown in Table 2. When the results were compared with internationally-published reference levels only levels of urinary $\mathrm{Al}$, As, $\mathrm{Mn}$ and $\mathrm{Si}$ were elevated. Urinary levels $\mathrm{Se}$ and $\mathrm{Cu}$ were lower than the published referenced ranges. No differences were observed between the two study visits in urinary concentrations of $\mathrm{Cd}, \mathrm{Pb}$ and $\mathrm{Hg}$ but a decrease in the median levels of $\mathrm{Al}, \mathrm{As}, \mathrm{Cr}, \mathrm{Co}, \mathrm{Cu}, \mathrm{Mn}, \mathrm{Se}$ and $\mathrm{Sr}$ urinary concentrations was observed at visit 2 compared to visit 1 (Table 2). When comparing men whose kidney function remained stable over the follow-up period and those whose kidney function declined (Figure 1), no differences were observed for any of the metal concentrations. An increase in $\mathrm{Pb}$ was noted in the three women with declining kidney function (Supplementary Figure 1). When values uncorrected for creatinine were examined, the levels of $\mathrm{As}, \mathrm{Cd}, \mathrm{Pb}$ and $\mathrm{Hg}$ were found to be slightly increased for both men and women at visit 2 but again no differences were observed between the kidney function trajectory groups (Supplementary Figure 2 and 3).

In the subset of samples analysed for pesticides, the highest median creatinine-adjusted urinary concentrations were found for 2,4-D, 3-PBA, DCCA, ETU, TEB-OH and TCP (Table 3). Glyphosate urinary concentrations were below the limit of detection (LOD) $(0.1 \mathrm{ng} / \mathrm{mL})$ for the majority of the samples (68\% and $70 \%$ of all samples tested at visit 1 and visit 2 , respectively). 4F3PBA, CFCA, MCPA, OH-PYM and 5-OH-TBZ were below the LOD $(0.1 \mathrm{ng} / \mathrm{mL}$ and $0.5 \mathrm{ng} / \mathrm{mL}$ for 5-OH-TBZ). When comparing those whose kidney function remained stable over the follow-up period and those whose kidney function declined, no differences were observed for concentrations of any of the pesticides or metabolites in men (Table 3 and Figure 2) or women (Table 3 and Supplementary Figure 4). Between the two visits, there was a decrease in the log transformed creatinine-corrected 
concentration of 2,4-D, 3-PBA, DCCA, ETU, TEB-OH and TCP in men (Figure 2). When examining values uncorrected for creatinine (Supplementary Figure 5), 2,4-D and TCP remained lower at visit 2 but again no differences were seen between renal function trajectory groups. Similarly, in the small number of women, there was a decrease in the log transformed creatinine-corrected and uncorrected concentrations of, 3-PBA, ETU, TEB-OH and TCP at visit 2 (Supplementary Figure 6).

OTA and CIT were below the LOD $(\leq 0.82 \mathrm{nmol} / \mathrm{L})$ for both analytes and no further analyses were performed.

\section{Discussion}

\section{Key Results}

This study tested the hypotheses that exposure to metals and metalloids, pesticides and mycotoxins play a primary causative role in the development of MeN. Although urinary levels of $\mathrm{Al}, \mathrm{As}, \mathrm{Mn}$ and Si were above published reference values, and evidence of exposure to pesticides was found, there were no associations with decline of kidney function. OTA and CIT levels were below the LOD excluding them as potential causative factors of MeN.

These data suggest significant environmental exposure to Al and total As (although we were unable to speciate As, see below). Median levels of Al and total As for both men and women at visit 1 and visit 2, were higher than the reference ranges reported for European and Mexican American populations, between 4 and 7 times for $\mathrm{Al}$ and between 6 and 9 times for total As, respectively (18-20). Although systemic symptoms of acute $\mathrm{Al}$ and As toxicity can be non-specific (e.g. headache and nausea), characteristic manifestations (e.g. altered mental state, hyperpigmentation, keratosis, melanosis or nail changes) (28) were not reported by members of the cohort. High levels of metals (e.g. As or Cd) can induce acute kidney injury and have been proposed as biomarkers of kidney injury of other types, however it is unclear if, or at what levels, these metals cause chronic kidney injury (29).

Population-level urinary As is highly variable, and lack of speciation limits the inferences that can be drawn. As is present in various pesticides and use and/or unsafe storage of these chemicals could lead 
to direct exposure of workers and family members or to water and soil contamination. Fish is the main dietary source of As, and furthermore, Nicaragua is a volcanic country potentially increasing the population's exposure to metals, through contaminated water, soil or air (30). A study measuring As in 19 workers with MeN showed As excretion in the normal range (6) but other published data from 99 sugarcane workers in north-western Nicaragua reported that the eGFR of the group with the most elevated concentrations of As in urine (above $90^{\text {th }}$ percentile) was $9 \mathrm{ml} / \mathrm{min} / 1.73 \mathrm{~m}^{2}$ lower than workers with low urinary As levels. (31).

In our study, a decrease in the median concentration of creatinine-corrected $\mathrm{Al}, \mathrm{Cr}, \mathrm{Co}, \mathrm{Cu}, \mathrm{Mn}, \mathrm{Se}$ and $\mathrm{Sr}$ was observed between visit 1 and visit 2 . This may be due to changes in metal or chemical concentrations in water sources at different times of the year. It is also possible that this relates, at least in part, to creatinine correction as these differences were no longer apparent when uncorrected levels were examined as median levels of creatinine in the samples from visit 2 were relatively higher than visit 1 . In turn, this increase in urinary creatinine might result from urinary concentration (due to hotter weather and more sweating), increased creatinine production (e.g. due to increased protein intake during the harvesting) or both.

Non-occupational exposure to low doses of pesticides occurs widely from residues in food and/or contaminated air and water (32). Pesticides are widely used in Nicaragua to increase crop quality and yield. In Nicaragua pesticide use doubled between 2000 and 2004 (17). In the sugarcane industry, herbicides are the most used pesticides, and a mixture of 2,4-D, terbutryn, and ametryn is commonly used in the sugar production process in the study area (33). Subsistence farmers use organophosphate and pyrethroid insecticides (included in this study), as well as paraquat $(34,35)$. Detectable levels of 2,4-D, 3-PBA, DCCA, ETU, TEB-OH and TCP were found in the majority of samples tested from both the stable and the declining kidney function groups. Overall, the measured concentrations of pesticide metabolites, suggest exposure to 2,4-D, chlorpyrifos of which TCP is a metabolite; and pyrethroids (such as cypermethrin of which 3-PBA and DCCA are metabolites). Although glyphosate has been proposed to play an important role in the development of $\mathrm{CKDu}(36)$, the urinary levels of excreted glyphosate in the present study were below the LOD in the majority of the samples. In 
contrast to the results from metals, using raw values rather than creatinine adjustment did not substantially alter the differences in pesticide and pesticide metabolite concentrations between the two visits.

Finally, the absence of urinary OTA and CIT make it unlikely that contamination of food with these fungal toxins is a cause of MeN in Nicaragua. This contrasts with findings from other endemic areas with CKDu, in particular Tunisia, where a high prevalence of OTA in serum has been related to the development of CKD (15).

\section{Strengths and limitations}

This study is the first to systematically analyse urine samples for various potential nephrotoxic factors before and after the sugarcane harvest using longitudinal outcome data in a Nicaraguan population at risk of developing MeN. Previous studies generally used a cross-sectional design which is prone to reverse causation and restricts cases to the late stages of disease (to prevent misclassification) making it even more challenging to draw conclusions regarding causation. Furthermore, the community-based nature of this study means the exposures in the entire at-risk population are assessed with lower risk of selection bias.

However, some limitations of the present study should be noted. Our study is only moderately sized, although the nature of the disease and its high prevalence in this population means that the power to detect associations with the outcome (rapid decline in eGFR) is higher than many studies with a similar number of participants. Post-hoc power calculations suggested it would be possible to detect associations with clinically important increases for most of the urinary metal concentrations. For example, we estimate there would have been over $80 \%$ power to detect a difference in As concentrations of $3 \mu \mathrm{mol} / \mathrm{mol}$ creatinine between groups. Similar calculations suggested we would have had $80 \%$ power to detect a difference in log transformed creatinine corrected levels of any of the agrochemicals of 0.8 standard deviations (SD). Clinically-relevant increases in urinary pesticide metabolites and OTA or CIT concentrations have not been defined, but it seems likely we would have 
detected biologically important differences between the kidney function trajectory groups if there was a true association.

A key limitation of this work is that exposures were only measured twice, once at baseline and again 6-months later (each side of the main harvest), an approach which although likely to capture those with highest overall ambient exposures but importantly may misclassify those with high but intermittent, e.g. occupational, exposure. However, comprehensive assessment of time-accumulated pesticide exposure would require a biomonitoring study conducted over prolonged periods or the use of other approaches to assess retrospective pesticide exposures which were beyond the scope of this work. Furthermore, we analysed only urine samples when for some analytes other biosamples (e.g. whole blood or hair) may better reflect exposure.

In addition, the non-speciation of urinary As, restricts our discussion about potential sources of As exposure. Finally, the limited sample size means it is not possible to investigate interactions between the measured exposures and other risk factors (e.g. dehydration/heat or genetic predisposition) that might underlie the evolution of disease.

\section{Conclusions}

$\mathrm{MeN}$ is still causing the death of tens of thousands rural workers in Central America. Although many hypotheses have been proposed, the exact cause(s) of the disease remain to be established. Our findings suggest that exposure to OTA and CIT is not a cause of MeN in Nicaragua. Similarly, higher levels of the metals and pesticide metabolites tested were not associated with renal function decline providing some evidence against a causal role in disease. However, high but intermittent exposure to the measured substances, other agrochemicals of widespread use that have not been quantified in the present study and interactions between the exposures measured and other factors, remain potential causes of MeN. 


\section{Tables}

\begin{tabular}{|c|c|c|c|c|c|c|c|c|c|}
\hline \multirow[t]{4}{*}{ Variables } & & \multicolumn{8}{|c|}{ Sex } \\
\hline & \multirow{3}{*}{ Categories } & \multicolumn{4}{|c|}{ Men } & \multicolumn{4}{|c|}{ Women } \\
\hline & & \multicolumn{2}{|c|}{ Visit $1(n=263)$} & \multicolumn{2}{|c|}{ Visit $2(n=213)$} & \multicolumn{2}{|c|}{ Visit $1(n=87)$} & \multicolumn{2}{|c|}{ Visit $2(n=78)$} \\
\hline & & $\mathbf{n}$ & $\%$ & $\mathbf{n}$ & $\%$ & $\mathbf{n}$ & $\%$ & $\mathbf{n}$ & $\%$ \\
\hline \multirow{2}{*}{ Age (years) } & $\leq 25$ & 168 & 63.9 & 128 & 60.1 & 51 & 58.6 & 37 & 47.4 \\
\hline & $>25$ & 95 & 36.1 & 85 & 39.9 & 36 & 41.4 & 41 & 52.6 \\
\hline \multirow{7}{*}{$\begin{array}{c}\text { Current } \\
\text { occupation }\end{array}$} & Sugarcane work & 50 & 19.0 & 104 & 48.8 & 10 & 11.5 & 9 & 11.5 \\
\hline & Agriculture work & 118 & 44.9 & 54 & 25.4 & 6 & 6.9 & 6 & 7.7 \\
\hline & Banana work & 13 & 4.9 & 15 & 7.0 & 1 & 1.2 & 3 & 3.9 \\
\hline & Commerce-related jobs & 5 & 1.9 & 14 & 6.6 & 9 & 10.3 & 7 & 9.0 \\
\hline & Construction & 10 & 3.8 & 6 & 2.8 & 0 & 0.0 & - & - \\
\hline & Other occupations & 14 & 5.3 & 10 & 4.7 & 3 & 3.4 & 4 & 5.1 \\
\hline & Economically inactive population & 53 & 20.2 & 10 & 4.7 & 58 & 66.7 & 49 & 62.8 \\
\hline \multirow{3}{*}{ Water supply } & Tap water & 139 & 52.9 & - & - & 47 & 54.0 & - & - \\
\hline & Dug well & 98 & 37.3 & - & - & 28 & 32.2 & - & - \\
\hline & Drilled well & 26 & 9.9 & - & - & 12 & 13.8 & - & - \\
\hline \multirow{2}{*}{ Smoking status } & Smoker & 103 & 39.1 & 98 & 46.0 & 1 & 1.2 & 1 & 1.3 \\
\hline & Non-smoker & 160 & 60.8 & 115 & 54.0 & 86 & 98.9 & 77 & 98.7 \\
\hline \multirow{2}{*}{$\begin{array}{c}\text { Alcohol } \\
\text { consumption }\end{array}$} & Any & 141 & 53.6 & 114 & 53.5 & 8 & 9.2 & 6 & 7.7 \\
\hline & None & 122 & 46.3 & 99 & 46.5 & 79 & 90.8 & 72 & 92.3 \\
\hline \multirow{2}{*}{$\begin{array}{c}\text { Pesticide } \\
\text { application }\end{array}$} & Yes & 135 & 51.3 & 63 & 29.6 & 12 & 13.8 & 12 & 15.4 \\
\hline & No & 128 & 48.7 & 150 & 70.4 & 75 & 86.2 & 66 & 84.6 \\
\hline
\end{tabular}

Table 1. Selected sociodemographic variables of the cohort stratified by sex at visit 1 (pre-harvest) and visit 2 (post-harvest). In total, 350 participants were recruited of who $16.9 \%$ lost to follow-up at visit 2. 


\begin{tabular}{|c|c|c|c|c|}
\hline \multirow{3}{*}{$\begin{array}{l}\text { Metals and metalloids } \\
(\mu \mathrm{mol} / \mathrm{mol} \text { creatinine })\end{array}$} & \multicolumn{4}{|c|}{ Sex } \\
\hline & \multicolumn{2}{|c|}{ Men } & \multicolumn{2}{|c|}{ Women } \\
\hline & Visit $1(n=263)$ & Visit $2(n=213)$ & Visit $1(n=87)$ & Visit $2(n=78)$ \\
\hline & Median $\left(25^{\text {th }}-75^{\text {th }}\right.$ IQR $)$ & Median $\left(25^{\text {th }}-75^{\text {th }}\right.$ IQR $)$ & Median $\left(25^{\text {th }}-75^{\text {th }}\right.$ IQR $)$ & Median $\left(25^{\text {th }}-75^{\text {th }} I Q R\right)$ \\
\hline $\mathrm{Al}$ & $360.6(216.5-629.2)$ & $232.7(157.4-393.9)$ & $384.9(222.8-631.2)$ & $330.3(170.6-534.1)$ \\
\hline Total As & $120.0(74.7-186.2)$ & $83.1(60.1-135.5)$ & $120.6(81.3-168.3)$ & $100.4(62.7-148.0)$ \\
\hline $\mathrm{Cd}$ & $0.3(0.2-0.5)$ & $0.3(0.2-0.4)$ & $0.4(0.2-0.6)$ & $0.3(0.2-0.5)$ \\
\hline $\mathrm{Cr}$ & $0.8(0.8-1.4)$ & $0.7(0.5-1.6)$ & $0.9(0.6-1.6)$ & $0.9(0.6-1.9)$ \\
\hline $\mathrm{Co}$ & $0.9(0.7-1.4)$ & $0.8(0.6-1.7)$ & $1.2(0.8-1.8)$ & $1.0(0.7-2.0)$ \\
\hline $\mathrm{Cu}$ & $16.0(12.3-21.8)$ & $12.5(10.0-16.4)$ & $14.0(12.2-22.5)$ & $13.2(9.8-18.2)$ \\
\hline $\mathrm{Pb}$ & $0.3(0.2-0.4)$ & $0.3(0.2-0.5)$ & $0.3(0.2-0.4)$ & $0.3(0.2-0.5)$ \\
\hline $\mathrm{Mn}$ & $3.3(1.6-6.4)$ & $2.5(1.4-5.6)$ & $3.9(2.3-7.0)$ & $4.1(2.6-7.6)$ \\
\hline $\mathrm{Hg}$ & $0.1(0.05-0.14)$ & $0.1(0.03-0.14)$ & $0.1(0.05-0.23)$ & $0.1(0.05-0.2)$ \\
\hline $\mathrm{Se}$ & $23.9(18.8-32.7)$ & $19.8(15.5-26.8)$ & $27.1(21.1-35.1)$ & $23.7(18.5-29.2)$ \\
\hline $\mathrm{Si}$ (mmol/mol creatinine) & $179.9(110.2-276.2)$ & $165.6(118.1-234.1)$ & $152.9(77.4-207.0)$ & $147.1(108.2-219.1)$ \\
\hline $\mathrm{Sr}$ & $282.8(121.4-627.0)$ & $191.7(137.7-261.5)$ & $200.1(84.0-437.9)$ & $153.8(111.3-237.0)$ \\
\hline
\end{tabular}

Table 2. Creatinine-adjusted urinary concentrations of metals and metalloids in men and women at visit 1 (pre-harvest) and visit 2 (post-harvest)

Described as median and interquartile range (IQR). See text for abbreviations. 


\begin{tabular}{|c|c|c|c|c|c|c|}
\hline \multirow{3}{*}{$\begin{array}{c}\text { Pesticides and metabolites } \\
(\mu \mathrm{mol} / \mathrm{mol} \text { creatinine })\end{array}$} & \multicolumn{4}{|c|}{ Sex } & \multirow{2}{*}{\multicolumn{2}{|c|}{$\begin{array}{c}\text { Samples } \geq \text { LOD } \\
\text { Whole cohort } \\
\end{array}$}} \\
\hline & \multicolumn{2}{|c|}{ Men } & \multicolumn{2}{|c|}{ Women } & & \\
\hline & Visit $1(n=50)$ & Visit $2(n=40)$ & Visit $1(n=7)$ & Visit $2(n=6)$ & Visit $1(n=57)$ & Visit $2(n=46)$ \\
\hline & $\begin{array}{c}\text { Median }\left(25^{\text {th }}-75^{\text {th }}\right. \\
\text { IQR) }\end{array}$ & $\begin{array}{c}\text { Median }\left(25^{\text {th }}-75^{\text {th }}\right. \\
\text { IQR })\end{array}$ & $\begin{array}{c}\text { Median }\left(25^{\text {th }}-75^{\text {th }}\right. \\
\text { IQR })\end{array}$ & $\begin{array}{c}\text { Median }\left(25^{\text {th }}-75^{\text {th }}\right. \\
\text { IQR })\end{array}$ & $\%$ & $\%$ \\
\hline 2,4-D & $1.0(0.3-2.5)$ & $0.3(0.1-0.7)$ & $0.1(<$ LOD-1.1) & $0.2(<\mathrm{LOD}-0.8)$ & 91.2 & 91.3 \\
\hline 3-PBA & $0.9(0.6-1.7)$ & $0.7(0.3-0.8)$ & $2.0(0.5-2.2)$ & $1.0(0.6-2.3)$ & 100.0 & 100.0 \\
\hline 4F3PBA & $<\mathrm{LOD}$ & $<\mathrm{LOD}$ & $<\mathrm{LOD}$ & $<\mathrm{LOD}$ & 8.8 & 21.7 \\
\hline CFCA & $<\mathrm{LOD}$ & $<\mathrm{LOD}$ & $<\mathrm{LOD}$ & $<\mathrm{LOD}$ & 5.3 & 15.2 \\
\hline DCCA & $0.9(0.4-1.6)$ & $0.5(0.3-0.9)$ & $1.4(0.5-1.7)$ & $1.4(0.5-2.9)$ & 98.2 & 100.0 \\
\hline ETU & $0.7(0.2-1.6)$ & $0.4(0.2-0.7)$ & $0.4(0.2-1.7)$ & $0.3(0.2-2.6)$ & 92.9 & 100.0 \\
\hline Glyphosate & $0.0(<\mathrm{LOD}-0.1)$ & $0.0(<$ LOD-0.04) & $<\mathrm{LOD}$ & $0.0(<\mathrm{LOD}-0.1)$ & 31.6 & 30.4 \\
\hline MCPA & $<\mathrm{LOD}$ & $<$ LOD & $<\mathrm{LOD}$ & $<\mathrm{LOD}$ & 0.00 & 0.0 \\
\hline OH-PYM & $<\mathrm{LOD}$ & $<\mathrm{LOD}$ & $<\mathrm{LOD}$ & $<\mathrm{LOD}$ & 1.7 & 17.4 \\
\hline 5-OH-TBZ & $<\mathrm{LOD}$ & $<\mathrm{LOD}$ & $<\mathrm{LOD}$ & $<\mathrm{LOD}$ & 0.00 & 0.0 \\
\hline TEB-OH & $0.2(0.1-0.3)$ & $0.1(0.1-0.2)$ & $0.1(0.1-0.2)$ & $0.1(<\mathrm{LOD}-0.3)$ & 91.2 & 89.1 \\
\hline TCP & $0.5(0.3-1.1)$ & $0.1(<\mathrm{LOD}-0.2)$ & $0.5(0.2-0.7)$ & $0.0(<\mathrm{LOD}-0.2)$ & 98.2 & 93.5 \\
\hline
\end{tabular}

Table 3. Creatinine-adjusted urinary concentrations of pesticides and pesticide metabolites from a nested case-control men and women at visit 1 (preharvest) and visit 2 (post-harvest) described as median and interquartile range (IQR) alongside the proportion of study participants with samples above the limit of detection. See text for abbreviations. LOD of 2,4D, 3-PBA, 4F3PBA, CFCA, DCCA, ETU, glyphosate, MCPA, OH-PYM, TEB-OH and TCP: $0.1 \mathrm{ng} / \mathrm{mL}$. LOD of 5-OH-TBZ: $0.5 \mathrm{ng} / \mathrm{mL}$. 


\section{Acknowledgements}

The authors are grateful to the participants of the study, the study team in Nicaragua for sample collection and preparation and all those who contributed with ideas and the analyses of samples, Kate Jones, Elizabeth Leese, James Staff, Howard Mason and Shahwaiz Iqbal from HSE, Buxton, UK, Moosa Faniband from Lund University, Sweden, and Kristina Jakobsson from University of Gothenburg, Sweden.

\section{Footnotes}

Contributors: ETS, BC, MGQ, JN, JM, DN and NP conceived and designed the studies, ETS analysed the data, ETS, MGQ, BC, JM, CTM, CL, PA contributed to data collection. All authors were involved in writing the paper and approved the submitted version.

Funding: This study was supported by the Colt Foundation (Grant number CF/03/14). Additional support was received from the Dutch National Postcode Lottery providing additional funding through Solidaridad. Thanks are due for the financial support to CESAM (UID/AMB/50017/2019), to FCT/MEC through national funds, and the co-funding by the FEDER, within the PT2020 Partnership Agreement and Compete 2020.

Competing interests: None declared.

Ethics approval: All participants provided written informed consent. The study was approved by the bioethical review board at the Medical Faculty of UNAN-León (Ref: FWA00004523/IRB00003342), and the research ethics committee of the London School of Hygiene and Tropical Medicine (Ref: 8643) in 2014.

Provenance and peer review: Not commissioned; externally peer-reviewed. 


\section{References}

1. Wesseling C, Crowe J, Hogstedt C, Jakobsson K, Lucas R, Wegman DH. The epidemic of chronic kidney disease of unknown etiology in Mesoamerica: a call for interdisciplinary research and action. Am J Public Health 2013;103(11):1927-30.

2. Peraza S, Wesseling C, Aragon A, Leiva R, García-Trabanino RA, Torres C, et al. Decreased kidney function among agricultural workers in El Salvador. Am J Kidney Dis 2012;59(4):53140.

3. Ramirez-Rubio O, McClean MD, Amador JJ, Brooks DR. An epidemic of chronic kidney disease in Central America: an overview. J Epidemiol Community Health 2012;67(1):1-3.

4. Ganguli A. Uddanam Nephropathy/Regional Nephropathy in India: Preliminary Findings and a Plea for Further Research. Am J Kidney Dis 2016;68(2)344-8.

5. Epidemiology Unit of Ministry of Heatlh, Nutrition and Indidenous Medicine, World Health Organisation NSF of SL. Prevalence of Chronic Kidney Disease of unknown aetiology and its risk factors in the district of Anuradhapura. 2017.

6. Wijkström J, González-Quiroz M, Hernandez M, Trujillo Z, Hultenby K, Ring A, et al. Renal Morphology, Clinical Findings, and Progression Rate in Mesoamerican Nephropathy. Am J Kidney Dis 2017;69(5):626-636.

7. Almaguer M, Herrera R, Orantes C. Histopathology of chronic kidney disease of unknown etiology in Salvadoran agricultural communities. MEDICC Rev 2014;16(2):49-54.

8. Herrera R, Orantes CM, Almaguer M, Alfonso P, Bayarre HD, Leiva IM, et al. Clinical characteristics of chronic kidney disease of nontraditional causes in Salvadoran farming communities. MEDICC Rev 2014;16(2):39-48.

9. Soderland P, Lovekar S, Weiner DE, Brooks DR, Kaufman JS. Chronic Kidney Disease Associated With Environmental Toxins and Exposures. Adv Chronic Kidney Dis 2010;17(3):254-64.

10. World Health Organization. Principles and Methods for the Assessment of Nephrotoxicity Associated with Exposure to Chemicals/published under the joint sponsorship of the United Nations Environment Programme, the International Labour Organisation, and the World Health Organization, Geneva: World Health Organisation; 1991.

11. Valcke M, Levasseur M, Soares A, Wesseling C. Pesticide exposures and chronic kidney disease of unknown etiology: an epidemiologic review. Environ Health 2017;16(49):1-20.

12. Pfohl-Leszkowicz A, Manderville RA. Ochratoxin A: An overview on toxicity and carcinogenicity in animals and humans. Mol Nutr Food Res 2007;51(1):61-99.

13. Ostry V, Malir F, Ruprich J. Producers and important dietary sources of ochratoxin A and citrinin. Toxins (Basel) 2013;5(9):1574-86.

14. Malir F, Ostry V, Pfohl-leszkowicz A, Malir J, Toman J. Ochratoxin A : 50 Years of Research. Toxins (Basel) 2016;8(7): 191.

15. Zaied C, Bouaziz C, Azizi I, Bensassi F, Chour A, Bacha H, et al. Presence of ochratoxin A in Tunisian blood nephropathy patients. Exposure level to OTA. Exp Toxicol Pathol 2011;63(78):613-8.

16. González-Quiroz M, Camacho A, Faber D, Aragón A, Wesseling C, Glaser J, et al. Rationale, description and baseline findings of a community-based prospective cohort study of kidney function amongst the young rural population of Northwest Nicaragua. BMC Nephrol 
2017;18:16.

17. González-Quiroz M, Smpokou E-T, Silverwood RJ, Camacho A, Faber D, Garcia BLR, et al. Decline in Kidney Function among Apparently Healthy Young Adults at Risk of Mesoamerican Nephropathy. J Am Soc Nephrol 2018;29(8):2200-2212.

18. Hoet P, Jacquerye C, Deumer G, Lison D, Haufroid V. Reference values and upper reference limits for 26 trace elements in the urine of adults living in Belgium. Clin Chem Lab Med 2013;51(4):839-49.

19. Morton J, Tan E, Leese E, Cocker J. Determination of 61 elements in urine samples collected from a non-occupationally exposed UK adult population. Toxicol Lett. 2014;231(2):179-93.

20. Centers for Disease Control and Prevention. Fourth Report on Human Exposure to Environmental Chemicals, 2009. Atlanta, GA: U.S. Department of Health and Human Services, Centers for Disease Control and Prevention.

21. Sieniawska CE1, Jung LC, Olufadi R, Walker V. Twenty-four-hour urinary trace element excretion: reference intervals and interpretive issues. Ann Clin Biochem. 2012;49(Pt 4):341-51.

22. Ekman E, Faniband MH, Littorin M, Maxe M, Jönsson BAG, Lindh CH. Determination of 5hydroxythiabendazole in human urine as a biomarker of exposure to thiabendazole using LC/MS/MS. J Chromatogr B 2014;973:61-7.

23. Ekman E, Maxe M, Littorin M, Jönsson BAG, Lindh CH. High-throughput method for the analysis of ethylenethiourea with direct injection of hydrolysed urine using online on-column extraction liquid chromatography and triple quadrupole mass spectrometry. J Chromatogr $B$ 2013;934:53-9.

24. Jensen PK, Wujcik CE, Mcguire MK, Mcguire MA. Validation of reliable and selective methods for direct determination of glyphosate and aminomethylphosphonic acid in milk and urine using LC-MS / MS. J Environ Sci Health B 2016;51(4):254-9.

25. Vidal A, Claeys L, Mengelers M, Vanhoorne V, Vervaet C, Huybrechts B, et al. Humans significantly metabolize and excrete the mycotoxin deoxynivalenol and its modified form deoxynivalenol-3-glucoside within 24 hours. Sci Rep 2018;8(1):5255.

26. Szczesniewski A. Increased Throughput with Alternate Column Regeneration Using Analytical LC/MS/MS Method for 126 Drugs and Metabolites in Urine in Clinical Research. 2018. Agilent Technologies, Wood Dale, IL.

27. Barr DB, Wilder LC, Caudill SP, Gonzalez AJ, Needham LL, Pirkle JL. Urinary Creatinine Concentrations in the U.S. Population: Implications for Urinary Biologic Monitoring Measurements. Environ Health Perspect 2005;113(2):192-200.

28. Dani SU, Walter GF. Chronic arsenic intoxication diagnostic score (CAsIDS). J Appl Toxicol 2018;38(1):122-44.

29. Vervaet BA, D'Haese PC, Verhulst A. Environmental toxin-induced acute kidney injury. Clin Kidney J 2017;10(6):747-58.

30. Alan H. Welch, D.B. Westjohn DRH and RBW. USGS- Arsenic in ground water of the united states: occurrence and geochemistry. Groundwater 2000;38(4):589-604.

31. Mcclean M, Amador JJ, Laws R, Kaufman JS, Weiner DE, Marcell J, et al. Report: Biological Sampling Report: Investigating biomarkers of kidney injury and chronic kidney disease among workers in Western Nicaragua. 2012. Boston University School of Public Health.

32. Damalas CA, Eleftherohorinos IG. Pesticide exposure, safety issues, and risk assessment indicators. Int J Environ Res Public Health 2011;8(5):1402-19. 
33. Mcclean M, Laws R, Rubio OR, Brooks DR. Final Report: Industrial Hygiene/Occupational Health Assessment: Evaluating Potential Hazards Associated with Chemicals and Work Practices at the Ingenio San Antonio (Chichigalpa , Nicaragua). Boston University School of Public Health. 2010;1-148.

34. Wesseling C, Aragón A, González M, Weiss I, Glaser J, Rivard CJ, et al. Heat stress, hydration and uric acid: a cross-sectional study in workers of three occupations in a hotspot of Mesoamerican nephropathy in Nicaragua. BMJ Open 2016;6:e011034.

35. Teresa Rodriguez, Berna van Wendel de Joode, Christian H Lindh, Marianela Rojas, Ingvar Lundberg CW. Assessment of long-term and recent pesticide exposure among rural school children in Nicaragua. Occup Envir Med 2012;69:119-25.

36. Gunarathna S, Gunawardana B, Jayaweera M, Zoysa K. Glyphosate and AMPA of agricultural soil, surface water, groundwater and sediments in areas prevalent with chronic kidney disease of unknown etiology, Sri Lanka. J Environ Sci Health B 2018;53(11):729-737. 


\section{Figure Legends}

Figure 1. Selected log transformed creatinine-corrected urinary metal concentrations in men at visit 1 and visit 2 stratified by kidney function trajectory. A. Arsenic (As) levels. The dotted line represents the upper limits as characterised for a Mexican American population (20). $\mathrm{p}=0.92$ and $\mathrm{p}=0.89$ for differences between stable and declining groups using Student's t-test at visit 1 and visit 2 respectively. B. Cadmium (Cd) levels. The dotted line represents the upper limits as characterised for Belgian and Canadian populations $(18,19) \cdot \mathrm{p}=0.81$ and $\mathrm{p}=0.65$ for differences between stable and declining groups using Student's t-test at visit 1 and visit 2 respectively. C. Lead $(\mathrm{Pb})$ levels. The dotted line represents the upper limits as characterised for a Belgian population (18). $\mathrm{p}=0.31$ and $\mathrm{p}=0.43$ for differences between stable and declining groups using Student's t-test at visit 1 and visit 2 respectively.

D. Mercury (Hg) levels. The dotted line represents the upper limits as characterised for a Belgian population (18). $\mathrm{p}=0.19$ and $\mathrm{p}=0.60$ for differences between stable and declining groups using Student's t-test at visit 1 and visit 2 respectively. Number of samples from visit 1: 263 and visit 2: 213, of which 25 at each visit belong to the declining eGFR trajectory group.

Figure 2. Selected log transformed creatinine-corrected urinary pesticide and pesticide metabolites concentrations in men at visit 1 and visit 2 stratified by kidney function trajectory. A. 2,4 D levels. $\mathrm{p}=0.58$ and $\mathrm{p}=0.59$ for differences between stable and declining groups using Student's t-test at visit 1 and visit 2 respectively. B. Levels of 3-PBA, metabolite of various pyrethroids. $p=0.14$ and $\mathrm{p}=0.08$ for differences between stable and declining groups using Student's t-test at visit 1 and visit 2 respectively. C. Levels of DCCA, metabolite of various pyrethroids. $p=0.29$ and $p=0.33$ for differences between stable and declining groups using Student's t-test at visit 1 and visit 2 respectively. D. Levels of TCP, metabolite of the organophosphate chlorpyrifos. $p=0.57$ and $p=0.22$ for differences between stable and declining groups using Student's t-test at visit 1 and visit 2 respectively. Number of samples at visit 1:50 and visit 2:40, of which 25 at each visit belong to the declining eGFR trajectory group. 